\title{
VIRTUAL CONNECTION BETWEEN DIGITAL AND PHYSICAL WORLD BASED ON SIXTH SENSE TECHNOLOGY
}

\author{
Sneha Verma And S.Sugumaran \\ School of Electrical Electronics and Communication, Galgotias University, NCR, U.P.
}

\begin{abstract}
The Sixth Sense 'An Extrasensory Perception' has turned into the new called 'The Sixth Sense Technology' which has emerged in few years. Sixth Sense Technology is a mini-projector coupled with a camera and a cell phone-which acts as the computer and connected to the Cloud, all the information stored on the web. In this time we have seen lots of technology devices which are mind blowing inventions for human's. As a latest technology called six sense technologies. Six sense technology is a kind of device which does not need to carry any heavy technology device such as camera, watch \& computers etc. Here if we have to take picture of any beautiful things or any things then we do not need a camera.

Six sense technology is a new \& interesting type of technology, which is easiest using for all type of people. The main factor of six sense devices is the distance, distance between the gesture and camera neither very large nor very small. This technology is all about interacting to the digital world in most efficient \& direct way. Human has many type of senses to perceive the information around us whenever we come across a thing, a person or a place. That information helps us make judgment the appropriate action to be taken. But arguably, the most useful information that can help us take the right decision and judgments is not naturally cognoscible information with the help of our five senses, namely the data, information and knowledge that mankind has amalgamated but, rather it's the 'Sixth Sense Technology.' This journel focuses on and makes us aware with the sixth sense technology which provides an integration of the digital world with the physical world; it helps us understand how the sixth sense device had overpowered the five natural senses; it also pours light over its various applications, its security related issues and further implications.
\end{abstract}

KEYWORDS

Six sense technology, Hand gestures, c++, JAVA programing, Digital World, Camera, Smartphone, Projector, Mirror.

\section{INTRODUCTION}

'Sixth Sense' is a wearable gestural interface that augments the physical world around us with digital information and lets us use natural hand gestures to interact with that information. Every one of us is aware of the five basic senses - seeing, feeling, smelling, tasting and hearing. These senses have evolved through millions of years. When these senses are not able to do any type of thing then six sense technologies came and this is totally based on the Indian mythology. i.e. it just depend on our thinking, in this technology the information's are stored traditionally on a paper or a digital storage device. Sixth sense technology helps to bridge this gap between tangible and non-tangible world. Six sense devices contain pocket projector, camera, web enabled smart phone, and coloured markers which are used to track the hand gestures by the user hands. This six sense technology can also obey hand gestures in the infamous minority report. This is a magic each \& everywhere we want, it's a gateway between digital \& real world.

DOI :10.5121/ijcsity.2016.4101 


\section{WHAT IS SIX SENSE?}

Sixth Sense in scientific or non-scientific terms is defined as Extra Sensory Perception or in short ESP. It involves the reception of information not gained through any of the five senses. Nor is it taken from any experiences from the past or known. Sixth Sense aims to more seamlessly integrate online information and tech into everyday life. By making available information needed for decision-making beyond what we have access to with our five senses, it effectively gives users a sixth sense. [2]

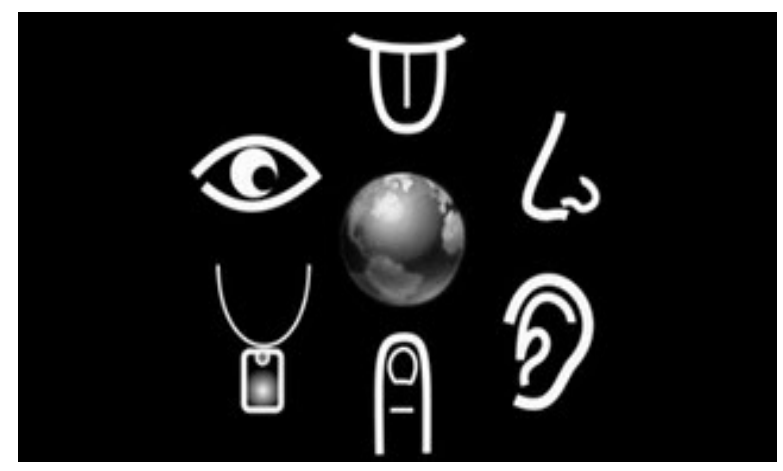

Figure no- 1 five senses

\section{Hardware Component Of Six Sense Device}

In six sense technology uses following devices - 1.camera 2.mobile component 3.mirror component 4.projector 5.colored marker.

3.1.Mobile component: - Consist of a web enabled smart phone which process the data send by the camera.

3.2.Camera: - It captures the image of the object in view \& tracks the users hand gestures. It sends data to smart phone for further processing. Basically it act as a digital eye which connect to the world of digital information.

3.3.Mirror component: - The uses of the mirror are significant as the projector, dangles pointing down wards from the neck.

3.4.Projector: - The projector is the key output device of the six sense system the projector visually augments surfaces walls and physical object the user is interacting with by projecting digital information and graphical user interface the projector itself contains a battery inside with three hours of battery life. Suggested product of projectors is LED or LASER.

3.5.Colored marker: - Color marker is used to marking the user's finger with color red, green, yellow \& blue. It's placed at the tip of user's fingers here web cam to recognize the hand gestures. [3] There is no boundation of taking a color, we can choose any color. 
International Journal of Computational Science and Information Technology (IJCSITY) Vol.4,No.1,February 2016

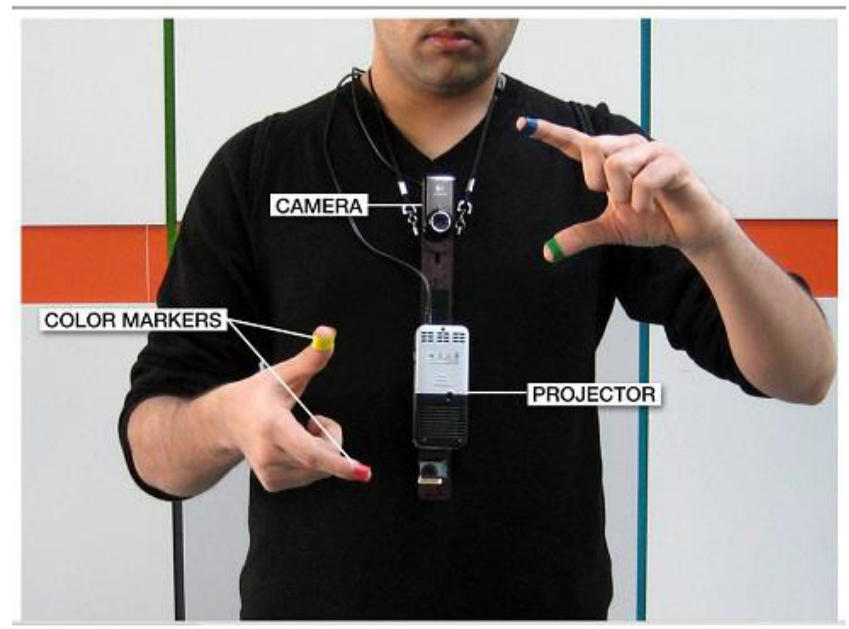

Figure no- 2 hardware component

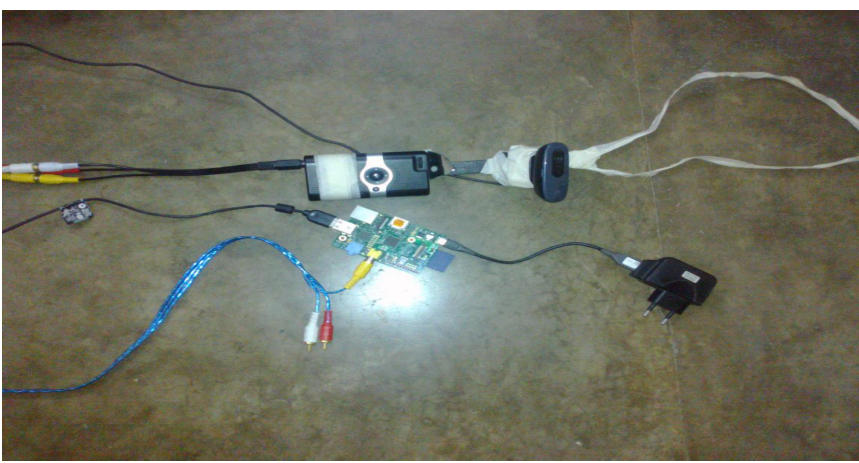

Figure no- 3 original device

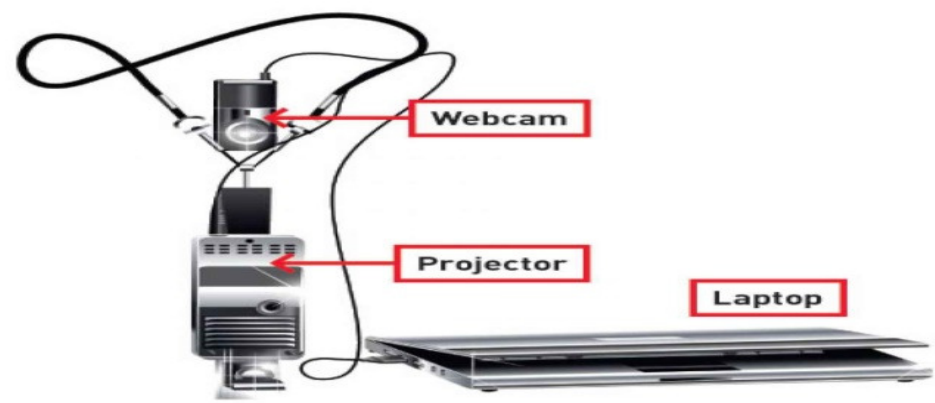

Figure no- 4 model of six sense devices

\section{WORKING OF SENSE DEVICES}

The sixth sense device uses different technologies like gesture recognition, image processing, etc. At present the commercial product is not launched but the prototype is prepared. The sixth sense prototype is made by using very common and easily available equipment's like pocket projector, 
a mirror, mobile components, color markers and a camera. The projector projects visual images on a surface. This surface can be wall, table, book or even your hand. Thus, the entire world is just like a screen now. When user moves their hands to form different movements with colored markers on the finger tips, the camera captures these movements. Both the projector and the camera are connected to the mobile computing device in the user's pocket. Recognition is made using computer vision technique. These markers act as visual tracking fiducials. The software program processes this video stream data and interprets the movements into gestures. The gestures are different from one another and are assigned some commands. These gestures can act as input to application which is projected by the projector. The projector is aligned downwards for compactness; therefore images would be formed at the user's feet if mirror wasn't used. The mirror reflects the image formed by the projector to front. The entire hardware is fabricated in the form of a pendent and helmet also. The entire product cost around $\$ 350$ and that also because of projector. It works very similar like a touch screen phone with entire world as the screen.

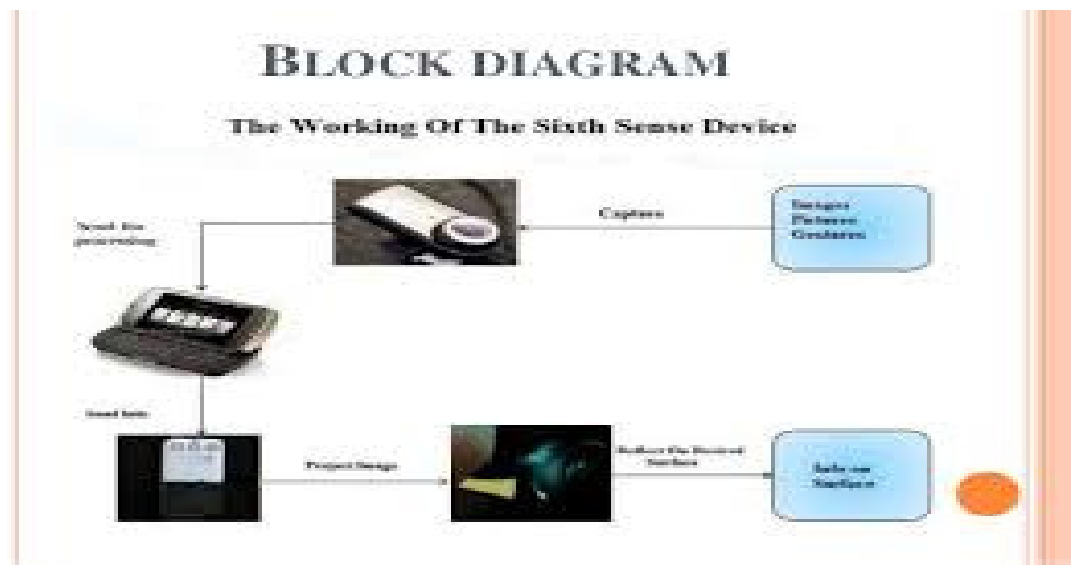

Figure-no 5 working diagram

\section{SOFTWARE USED}

Software program processes the video stream data captured by the camera, the Sixth Sense software will be open source. As far as this seems to be a little set of items, there will not be user interfaces or much advanced programs for the users. There will be much harder and secured coding inside the device to make sure the security of the software. It will be interesting to know the new language for coding for a Sixth Sense device, as for as this seems to be a little set of items there will not be user interfaces or much advances program for the users. There will be much harder \& secured coding inside the device to make sure the security of the software. The prototype system runs on windows platform \& majority of the

code is written in $\mathrm{C}++\& \mathrm{C \#}$. Uploading newer versions as it is being developed this will also include a mobile version. [4]

\section{WHY DISCOVER SIX SENSE TECHNOLOGY}

Basically humans take decisions after acquiring inputs from the senses. But the information is not sufficient in humans than its effect to the right decisions. But in this time with the help of Internet, we collect a huge number of information which making a good decision on a few minutes. This six sense technology provides us with the freedom of interacting with digital world 
with hand gestures .This technology has a wide application in the field of Artificial Intelligence. This methodology can aid in synthesis of bots that will be able to interact with humans. [5]

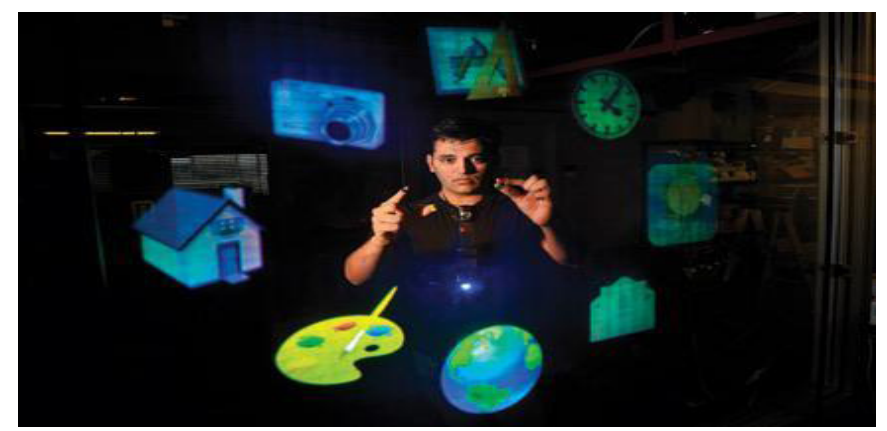

Figure no- 6 Internet access huge number of information

\section{BACKGROUND OF THIS TECHNOLOGY}

The history behind the six sense technology goes back into 1990s when Steve Mann first attempt to propose a neck worn projector \& a camera combination. The idea of implementing computer technologies to daily task as six senses was further developed by pranav mistry who also appears to be an MIT lab Phd student as well as Steve man. Pranav Mistry is an Indian computer scientist. At present, he is the head of Think Tank team Director and researcher of Samsung. He is best known for his work on six sense technology .[6] The first prototype of the six sense technology was actually bigger then what it looks like today \& it was not working properly to use in daily life. Pranav Mistry first tried out his idea on a simple computer mouse. First he put two rollers into one mouse and see if he could obtain data and guide the movements of the mouse. Then two rollers did not work properly so he decided to use four rollers and see if it could work better four rollers gave him the idea that he could use the same idea on fingers \& that is what the next moved on to. [7]

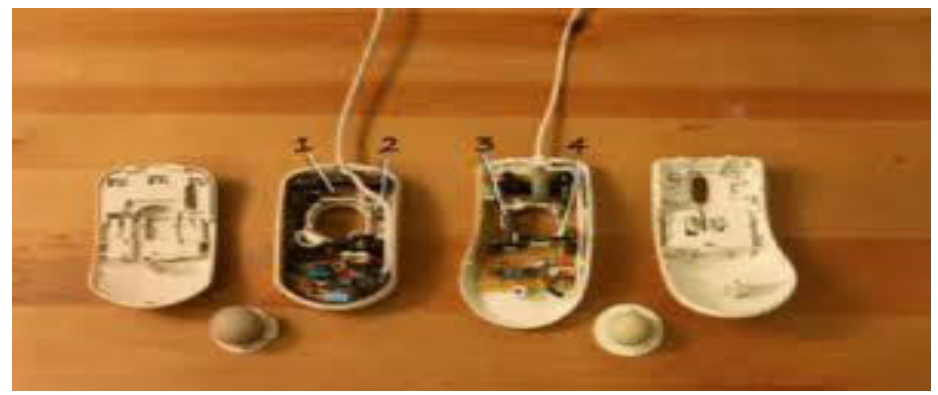

Figure no-7 mouse

\section{APPLiCATIONS}

\subsection{Making a call}

We can use the Sixth Sense technology to project a keypad onto your hand, and then use that virtual keypad to make a call. Calling a number also will not be a great task with the introduction of Sixth Sense Technology. No mobile device will be required, just type in the number with your 
palm acting as the virtual keypad. The keys will come up on the fingers. The fingers of the other hand will then be used to key in the number and call.

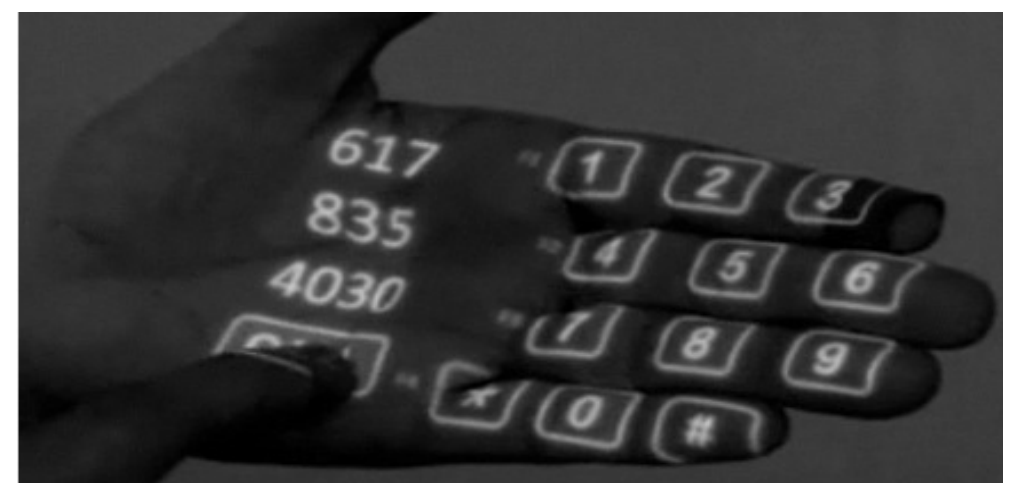

Figure no- 8 making a call

\subsection{Map Making}

The sixth sense also implements map which lets the user display the map on any physical surface and find his destination and he can use his thumbs and index fingers to navigate the map, for example, to zoom in and out and do other controls.

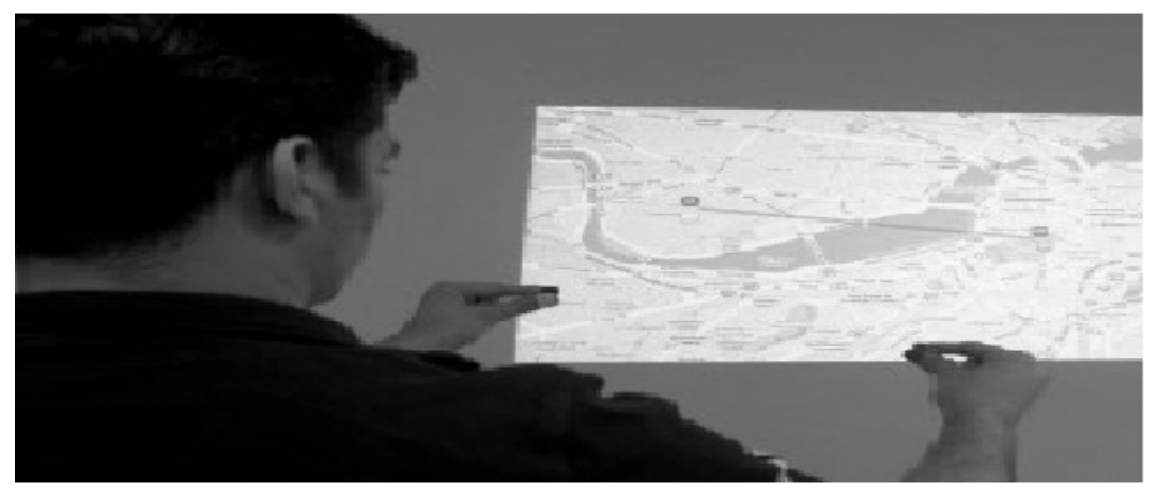

Figure no- 9 map making

\subsection{Making Virtual Wrist Watch}

Sixth Sense all we have to do is draw a circle on our wrist with our index finger to get a virtual Watch that gives us the correct time. The computer tracks the red marker cap or piece of tape, recognizes the gesture, and instructs the projector to flash the image of a watch onto his wrist. 


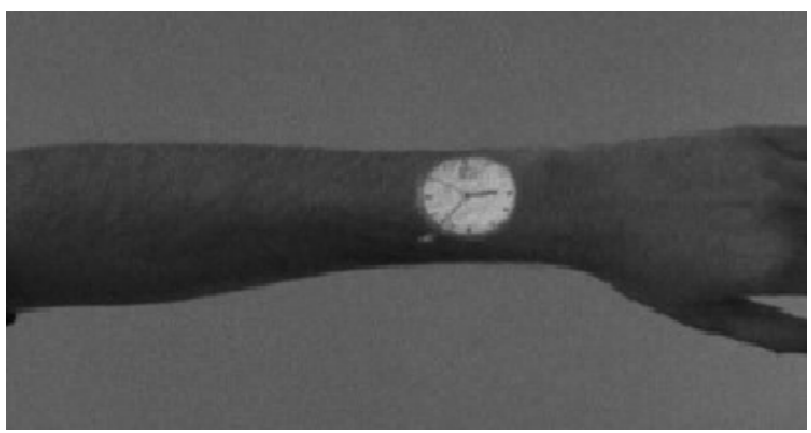

Figure no- 10 checking time in virtual wrist watch

\subsection{Create video on the paper}

The Sixth Sense system also augments physical objects the user is interacting with by projecting more information about these objects projected on them. For example, a newspaper can show live video news or dynamic information can be provided on a regular piece of paper. Thus a piece of paper turns into a video display.

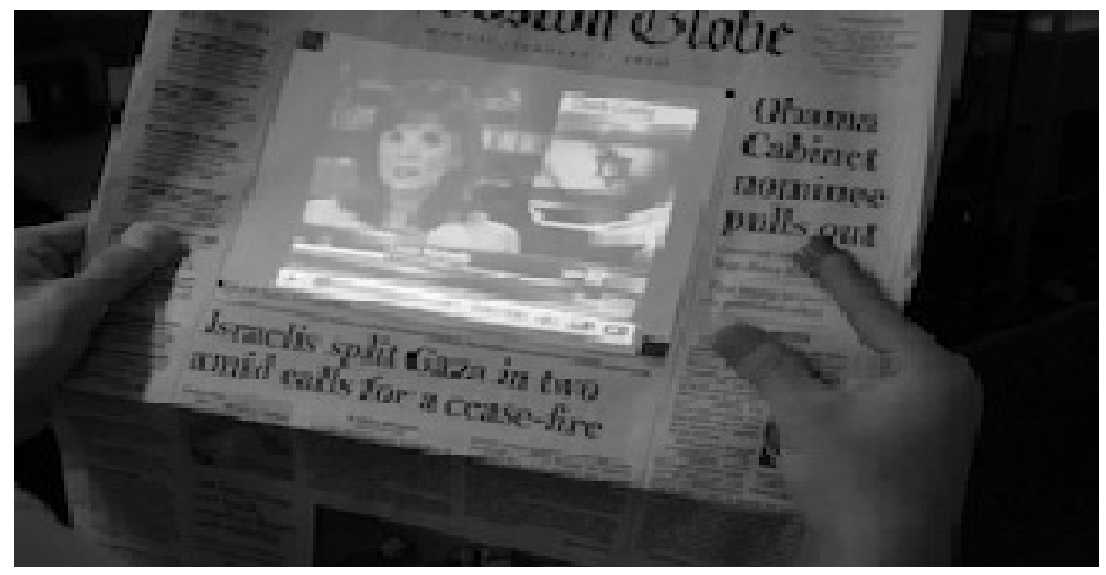

Figure no- 11 create multimedia reading

\subsection{Drawing On Desired Surface}

The drawing application lets the user draw on any surface by tracking the fingertip Movements of the user's index finger.

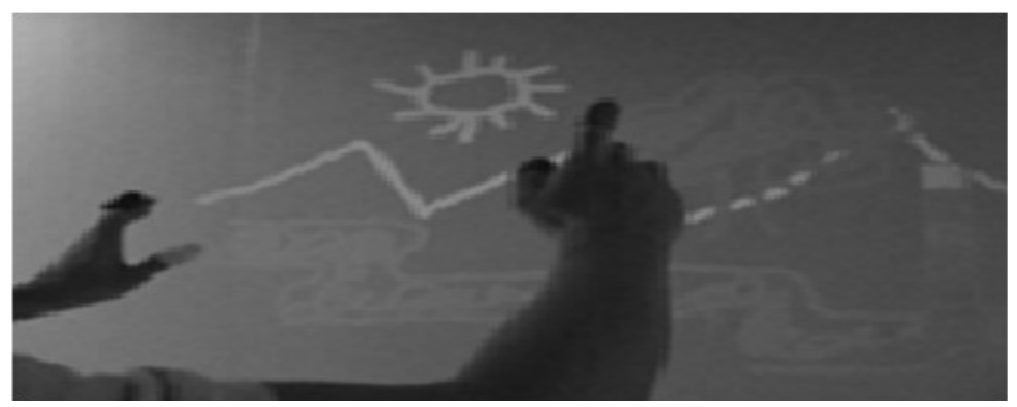

Figure no- 12 drawing 


\subsection{Zooming features}

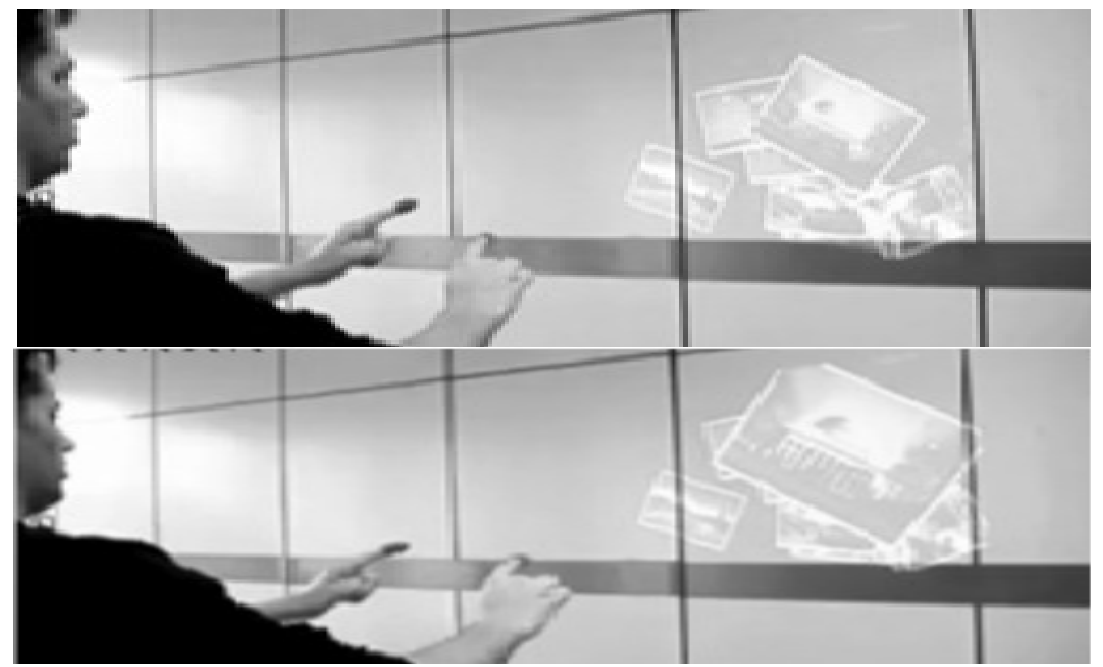

Figure no- 13 Zoom in and Zoom out

The user can zoom in or zoom out using intuitive hand movements.

\subsection{Get Product Information}

Maes says Sixth Sense uses image recognition or marker technology to recognize products you pick up, and then feeds you information on those products. For example, if you're trying to shop "green" and are looking for paper towels with the least amount of bleach in them, the system will scan the product you pick up off the shelf and give you guidance on whether this product is a good choice for you.

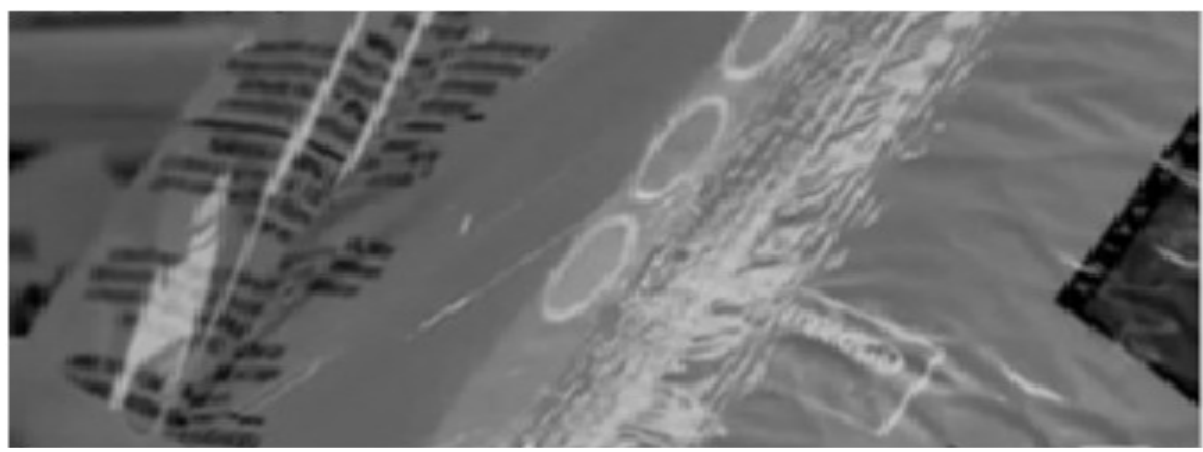

Figure no- 14 product information

\subsection{Getting book information}

Maes says Sixth Sense uses image recognition or marker technology to recognize products you pick up, and then feeds you information on books. The system can project AmazonRatings on that book, as well as reviews and other relevant information. 


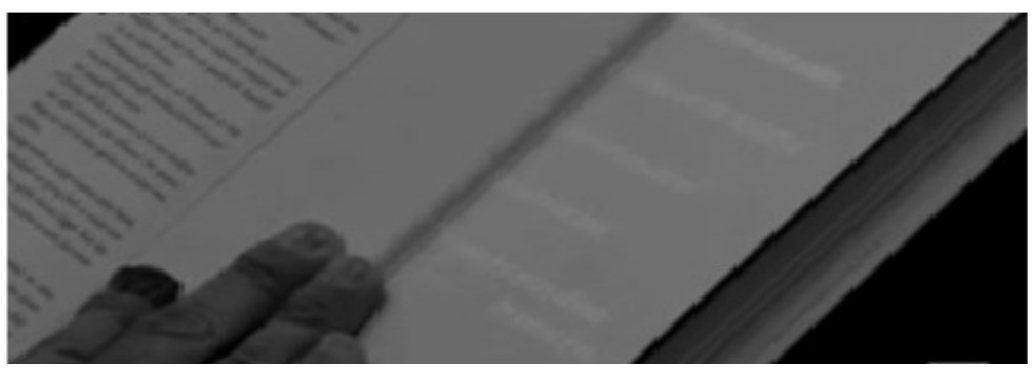

Figure no- 15 book information

\subsection{Taking a pictures}

If we fashion our index fingers and thumbs into a square (the typical "framing" gesture), the system will snap a photo. After taking the desired number of photos, we can project them onto a surface, and use gestures to sort through the photos, and organize and resize them with the help of zooming feature.

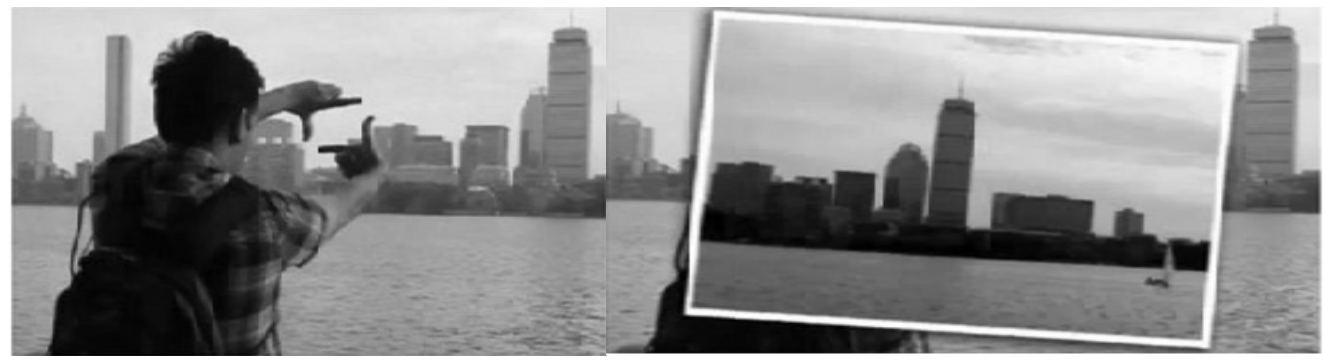

Figure no- 16 taking pictures

\subsection{Get Flight Updates}

The system will recognize your boarding pass and let you know whether your flight is on time and if the gate has changed.

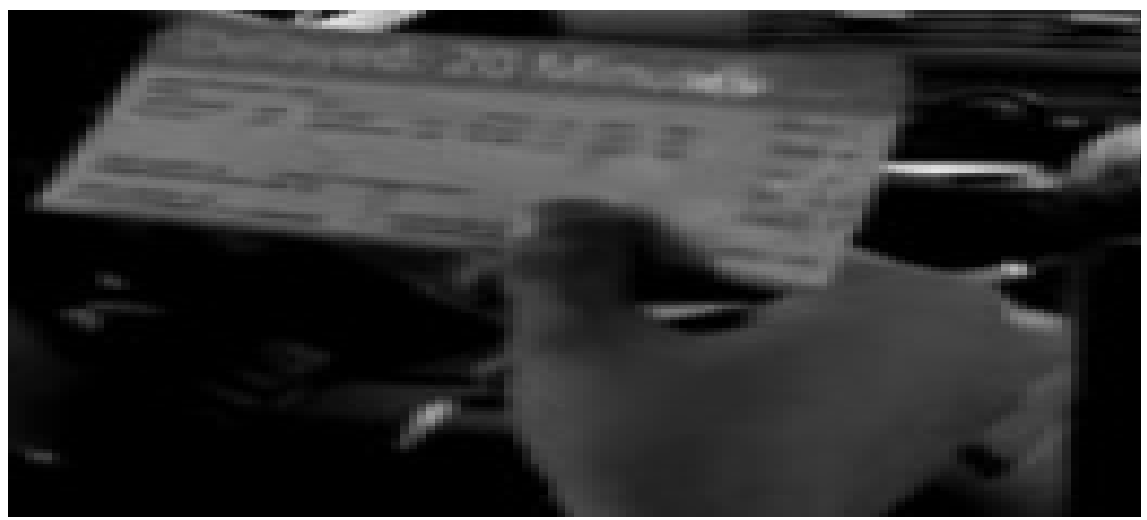

Figure no- 17 Flight updates 


\subsection{Getting The Of Information On People}

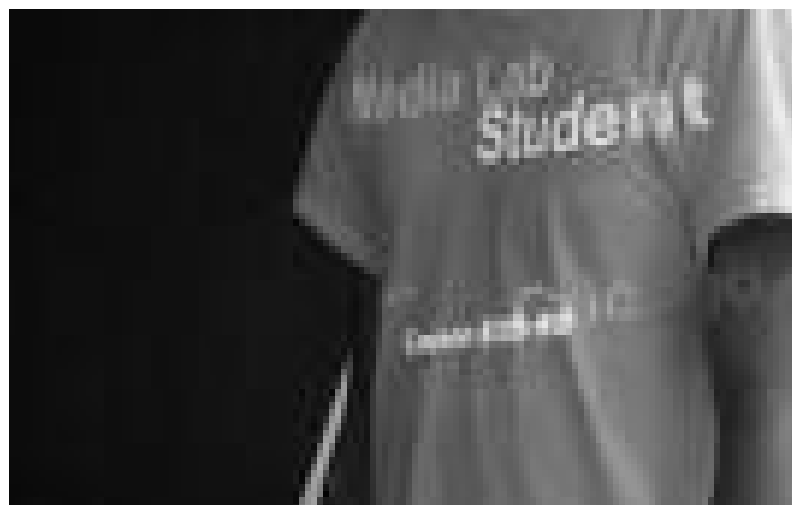

Figure no- 18 Information on people

Sixth Sense also is capable of "a more controversial use". When you go out and meet someone, projecting relevant information such as what they do, where they work, and also $\mathrm{m}$ it could display tags about the person floating on their shirt. It could be handy if it displayed their Facebook relationship status so that you knew not to waste your time.

\section{WAY FORWARD}

Upcoming projects making use of sixth sense technology.

\subsection{Mouse less}

Mouse less is a concept which is developed in order to avoid the usage of mouse in computers. It helps to provide a familiarity of the physical mouse without actually requiring a physical mouse. Even through the computer hardware technology has been developed to a greater extent, still there has been no proper substitute for a physical mouse. This new invention aims to remove the requirement of having a physical mouse altogether but still it provides the feeling of a physical mouse that users are familiar with. It basically consists of an infrared laser beam and infrared camera. Both laser and camera are embedded together with computer. The laser beam module is modified with a line cap and placed such that it creates a plane of IR laser just above the surface the computer sits on. The user cups their hand, as if a physical mouse was present underneath, and the laser beam lights up the hand which is in contact with the surface. The IR camera detects those bright IR blobs using computer vision. The change in the position and arrangements of these blobs are interpreted as mouse cursor movement and mouse clicks.

\subsection{Third eye}

Third eye is new technology that enables multiple viewers to see different things on a same display screen at the same time. With third eye, we can have a public sign board where a Japanese tourist sees all the instructions in Japanese and an American in English. We don't need to have the split screen in games now. Each player can see his/her personal view of game on the TV. screen. Two people watching TV can watch their favorite channel on a single TV. Screen. A public display can show secret messages or patterns. In the same movie theater, people can see different end of suspense movie. 


\subsection{Inktuitive}

Even though there has been a tremendous development of computer aided designing tools, still paper and pencil is the preferred by most of designers, especially in the earlier stages of designing. The project 'inktuitive' in aimed to combine the process of creation that is inherent in paper and pencil with the power of computing that the digital design tools provide. It also extends the natural work-practice of using physical paper by giving the pen the ability to control the design in physical three-dimensional space, freeing it from its tie to the paper. There always remains an intuition of pen and paper, but the lines that have been drawn are be captured and translated into shapes in the digital strokes. The platform provides a novel interaction mechanism for drawing and designing using above the surface pen movements. In other words 'inkuitive' can be considered as an intuitive physical design workspace that aims to bridge the gap and bring together the conventional design tools such as paper and pencil with the power and convenience of the digital tool for design.

\subsection{TaPuma}

TaPuMa is the short form of tangible public map. It is another attempt to bring the digital world nearer to the real world by exploring the techniques of six sense technology. Key word based search mechanism has vastly influenced and modified the ways in which we search information's. The keyword based search allows the user to filter out the required data from the vast data that are available on search engines such as yahoo and Google. TaPuMa allows people to use their own belongings, the objects they usually carry with them to access relevant and just-in-time information and locations of places from a public map. The project also explores and analyzes the advantages and challenges of this novel interface for information acquirement. The broad concept behind the project TaPuMa is objects Amelioration, where the functions of everyday objects can be expanded by using their affordances or functionalities in a variety of different contexts.

\section{CONCLUSION}

The sixth sense technology concept is an effort to connect this data in the digital world in to the real world. With the help or hand gestures we can draw any picture and zoom in or zoom out of that picture and another exciting application, when our Five senses not able to do anything then six sense arises, there are many application of six sense technology. the main thing of this technology, we can directly connect the digital world without help of any digital device, this technology makes a bridge between the physical world and digital world. The sixth sense technology and devices are definitely way forward .sixth sense is a quite efficient way compared to the text and graphic based user interface. It has the potential to form the transparent user interface for accessing the information around us. Even through the sixth sense devices are currently in development stages and have not been used in large now, it's quite predictable that this technology have the potential revolutionize the way in which we interacts with the digital word.

And there are another very exciting application of six sense technology to store a data in our fingertip, it will be possible with the help of six sense technology, memory card , P.D, is not necessary to store the data, we can easily store in our body and display on the desired surface. 


\section{ADVANTAGES}

1. Six sense technology is an open source.[7]

2. six sense technology is a gadget that could be work as jewellery \& can do various things like take pictures, given direction (like GPS) Browse the web etc.[1]

3. Camera can act as a eye for the blind person.[9]

4. Six sense technology is a user friendly device.

5. Six sense technology is so light, easy to use \& easy to implement

6. Six sense technology is not heavy to carry around

7. Six sense technology is not that expensive (cost about $\$ 350$ or $17000 /$-)

8. It uses hand gestures to interact with digital information.

9. Data access directly from machine in real time

10. It is gesture-controlled wearable computing device that feeds our relevant information and turns any surface into an interactive display.

\section{LIMITATION}

1. There is no easy way to fix if six sense device is break.

2. Six sense device cannot be used for long period of time

3. Privacy is a major issue; if we open up the gadget on a busy street then everyone would be able to see what we are doing

4. This device is not projecting a hard surface.

5. This technology is not attach to all the time with human body parts, because high waves radiation emitted may cause some disease

6. Projection is better in the night time $\&$ dark areas rather than mornings $\&$ bright areas.

7. Software does support the ability to use real time video streams in order to produce augmented reality

8. Hardware limitation of the devices that we currently carry around with us for example many phones will not allow the external camera feed to be manipulated in real time

9. Post processing can occur however [8]

\section{ACKNOWLEDGEMENT}

We would like to thank Dr. Sibaram Khara, Dean(Academics), Dr.Dilip Debnath, Dean, SEECE, Galgotias University, NCR, UP for their kind guidance and support in compilation of the paper.

\section{REFERENCES}

[1] Suryavanshi et al., International Journal of Advanced Research in Computer Science and Software Engg. 3(11), November - 2013, pp. 744-748

[2] www.blendernation.com/sixth-sense-technology/

[3] http:// dspace.cusat.ac.in

[4] http:// code.google.com/ six sense/ wiki/ hardware/software

[5] Www. Engineersgarage.com/articles/ six sense technology [6] en.wikipedia.org/ wiki/ pranav_mistry

[7] Misy 3310dogukan.weebly.com/ six sense history

[8] http:// six sense technology. hit103.blogspot.in

[9] www.scribd.com/ doc/ 29354104 / six sense technology.

[10] http://gizmodo.com/5167790/sixth-sense-technology-may-change-how-we-lookat-the-world-forever

[11] http://theviewspaper.net/sixth-sense-technology-will-revolutionize-the-world/ 


\section{Authors}

SNEHA VERMA is a student of GALGOTIAS UNIVERSITY, Greater Noida. She is currently pursuing M.Tech degree in Communication engineering. Her main areas of interest are optical Communication,computer networks and optical networks.

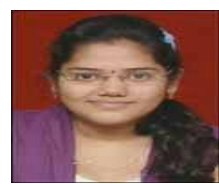

SUGUMARAN.S was born in Pallikonda,Vellore District, Tamil Nadu, India in 1976. He received his Bachelors of Engineering Degree from the Department of Electronics and Communication Engineering from Bharathiyar University, Coimbatore, India and Master's Degree in Optical Communication from CEG, Anna University, Chennai in 1998 and 2004 respectively. After working as Faculty in different designations in VIT University, Vellore, he is currently working as Assistant Professor Gr.-III in School of Electrical,Electronics and Communication Engineering, Galgotias University, Greater Noida,NCR. He is currently pursuing the $\mathrm{PhD}$ degree in Optical communication and networks. His research include nonlinear effects (FWM, CPM) on WDM networks and FTTH application . 